Diabetologe 2009 · 5:9-11

DOI 10.1007/s11428-008-0376-7

Online publiziert: 12. Januar 2009

(c) Springer Medizin Verlag 2009

\author{
W. A. Scherbaum ${ }^{1} \cdot$ W. Sandmann ${ }^{2}$ \\ ${ }^{1}$ Klinik für Endokrinologie, Diabetologie und Rheumatologie, WHO Collaborating \\ Centre for Diabetes, European Training Center in Endocrinology and Metabolism, \\ Universitätsklinikum Düsseldorf \\ ${ }^{2}$ Klinik für Gefäßchirurgie und Nierentransplantation, \\ Universitätsklinikum Düsseldorf
}

\title{
Periphere arterielle Verschlusskrankheit bei Diabetes
}

Die periphere arterielle Verschlusskrankheit (PAVK) ist eine häufig unterschätzte makrovaskuläre Komplikation des Diabetes. Es war uns daher wichtig, dieses Thema der überwiegend internistischen und hausärztlichen Leserschaft der Zeitschrift Der Diabetologe nahezubringen.

Ursächlich spielen bei der PAVK ähnliche Risikofaktoren eine Rolle, wie bei der koronaren Herzkrankheit und den zerebrovaskulären Verschlusserkrankungen, nämlich die arterielle Hypertonie, die Dyslipoproteinämie und das Rauchen. Bei Patienten mit einem Diabetes mellitus, speziell mit einem metabolischen Syndrom kommen diese Risikofaktoren gleich in Kombination daher, und der Diabetes verdoppelt oder verdreifacht das vorgenannte Risiko. Die PAVK hat beim Diabetiker einige Besonderheiten, die es zu berücksichtigen gilt; insbesondere ist dies ein häufiges Fehlen der Schmerzsymptomatik im Sinne einer Claudicatio intermittens, die rasche Progredienz der Arteriosklerose und das multilokuläre Befallsmuster: Nicht nur haben Patienten mit einer PAVK bei Diabetes zusäzlich eine KVK und/oder eine zerebrovaskuläre Verschlusskrankheit, sondern auch die peripheren Arterien, in der Regel der unteren Extremitäten, weisen im Gegensatz zu vielen Nichtdiabetikern meist multiple und langstreckige Stenosen auf. Wichtig ist zunächst, bei Patienten mit einem Diabetes auch an eine PAVK zu denken und unbedingt auch den Knöchel-Arm-Index dopplersonographisch zu bestimmen - auch wenn diese Bestimmung nicht separat abgerechnet werden kann. Professor Diehm vom Klinikum Karlsbad-Langensteinbach und seine Kollegen weisen in ihrem Artikel ausdrücklich darauf hin, dass die klinischen Basisuntersuchungen Inspektion, Palpation und Auskultation nicht ausreichen, um die Diagnose einer PAVK auszuschließen.

Nicht selten handelt es sich bei Patienten mit PAVK um ältere, kranke oder sogar kritisch kranke Menschen, die z. B. auch eine KHK oder eine eingeschränkte Nierenfunktion aufweisen, so dass eine Gefäßdarstellung mit jodhaltigen Röntgenkontrastmitteln und das damit verbundene Risiko einer Verschlechterung der Nierenfunktion nur zu vertreten ist, wenn sich daraus eine therapeutische Konsequenz ergeben kann. Hier ist eine interdisziplinäre Abstimmung bei den einzelnen diagnostischen und therapeutischen Schritten und bisweilen ein stufenweises Vorgehen angezeigt.

Professor Amendt vom Gefäßzentrum Oberrhein Mannheim-Speyer und seine Kollegen erläutern in ihrem Artikel die Möglichkeiten der konservativen Therapie, wobei - ähnlich wie bei der koronaren 


\section{Hier steht eine Anzeige.}

\author{
Springer
}

Herzkrankheit und der zerebrovaskulären Verschlusskrankheit die Lebensstil-Intervention eine zentrale Rolle einnimmt. Ziel ist eine Verbesserung der Lebensqualität des Patienten durch Steigerung der Gehfähigkeit und der allgemeinen Mobilität sowie durch Reduktion des Ischämieschmerzes. Die medikamentösen Behandlungsmöglichkeiten waren bis vor kurzem sehr begrenzt und wurden nun durch die Therapie mit Cilostazol, einem oral verabreichten selektiven Phosphodiesterase (PDE)-3-Inhibitor, bereichert.

Bei der symptomatischen PAVK, insbesondere aber beim Vorliegen eines diabetischen Fußsyndroms, reichen aber nichtinvasive Maßnahmen oft nicht aus, um die Durchblutungssituation an den Füßen so zu bessern, dass eine Heilung des Ulkus eintritt.

Frau PD Dr. Weis-Müller von der Klinik für Gefäßchirurgie und Nierentransplantation des Universitätsklinikums Düsseldorf und Kollegen zeigen hier eindrucksvolle eigene Ergebnisse der von der Gruppe um Herrn Prof. Sandmann durchgeführten operativen Revaskularisationen bei Patienten mit PAVK aus den Jahren 1996 bis 2005. Diabetiker waren hier weit überproportional vertreten. Die modernen operativen Methoden der peripheren Revaskularisation, z. B. mit Anlage eines kruropedalen Bypass, haben die Prognose beim gemischt neuropathischvaskulären diabetischen Fußsyndrom wesentlich verbessert. Die hier vorgestellten Daten zeigen, dass Nihilismus fehl am Platze ist. Wenn auch die Langzeitprognose der Diabetespatienten quoad vitam schlechter war als die der Nichtdiabetiker, so waren doch die Operationsergebnisse bei Diabetikern gleich gut, wie bei Nichtdiabetikern. Insbesondere wurde gezeigt, dass bei Patienten mit einem diabetischen
Fußsyndrom durch eine frühzeitige Revaskularisation kritisch durchblutungsgestörter Gliedmaßen evtl. in Kombination mit einer gewebesparenden Minoramputation auch bei amputationsgefährdeten Extremitäten eine hohe 5-Jahres-Beinerhaltungsrate erzielt werden konnte - ein Segen für die Lebensqualität der Betroffenen.

Wir würden uns freuen, wenn Sie von diesem Heft viele Anregungen für Ihre praktische Arbeit am Patienten mitnehmen könnten

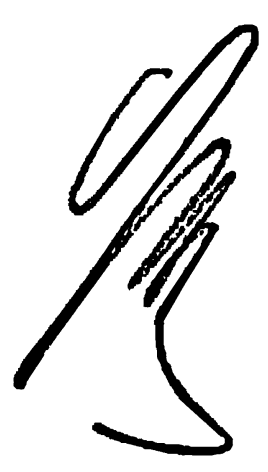

Wilhelm Sandmann

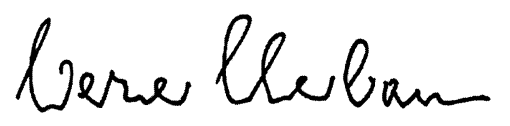

Werner Scherbaum

\section{Korrespondenzadresse}

Prof. Dr. W. A. Scherbaum

Klinik für Endokrinologie,

Diabetologie und Rheumatologie, WHO Collaborating Centre for Diabetes, European Training Center in Endocrinology and Metabolism,

Universitätsklinikum Düsseldorf Moorenstr. 5, 40225 Düsseldorf scherbaum@uni-duesseldorf.de 
Hier steht eine Anzeige.

算 Springer 